\title{
Strain-induced spin relaxation anisotropy in symmetric (001)-oriented GaAs quantum wells
}

\author{
D. J. English, P. G. Lagoudakis, and R. T. Harley \\ School of Physics and Astronomy, University of Southampton, Southampton SO17 1BJ, United Kingdom
}

P. S. Eldridge, ${ }^{*}$ J. Hübner, and M. Oestreich

Institute for Solid State Physics, Leibniz University of Hannover, Appelstrasse 2, D-30167 Hannover, Germany

(Received 18 August 2011; revised manuscript received 23 September 2011; published 31 October 2011)

\begin{abstract}
We show experimentally, using spin quantum beat spectroscopy, that strain applied to an undoped symmetric (001) GaAs/AlGaAs multiple quantum well causes an in-plane anisotropy of the spin-relaxation rate $\Gamma^{s}$, but leaves the electron Landé $g$ factor isotropic. The spin-relaxation-rate anisotropy gives a direct measure of the bulk inversion asymmetry and the strain contributions to the conduction-band spin splitting. The comparison of the measured strain-splitting coefficient $C_{3}$ for the quantum well with the value for bulk GaAs suggests a dependence on electron quantum confinement. The isotropic $g$ factor implies a symmetric conduction electron wave function, whereas the anisotropic spin-relaxation rate requires a nonzero expectation value of the valenceband potential gradient on the conduction-band states. Therefore, the experiment suggests that strain generates an effective valence-band potential gradient, while the conduction-band potential remains symmetrical to a good approximation.
\end{abstract}

DOI: 10.1103/PhysRevB.84.155323

PACS number(s): 71.70.Ej, 73.21.Fg

\section{INTRODUCTION}

The manipulation and control of conduction electron spins in semiconductors is of fundamental importance in spintronics. The application of an external magnetic field provides a direct method to reorient the spins but is cumbersome and therefore other methods are under active investigation. ${ }^{1,2}$ In III-V materials, the control of electron spin without the application of an external magnetic field is, in principle, possible via the spinorbit interaction, whereby an electron moving in the inversionasymmetric environment of the structure senses an effective magnetic field, which causes precession or reorientation of its spin. ${ }^{3,4}$ The effective magnetic field can be specified by a precession vector $\boldsymbol{\Omega}(\mathbf{p})$, whose frequency and direction depend on the electron's momentum vector $\mathbf{p}$.

In a heterostructure based upon III-V compounds, there can be several contributions to $\boldsymbol{\Omega}(\mathbf{p})$ corresponding to different sources of inversion asymmetry. ${ }^{3,5}$ The intrinsic inversion asymmetry of the zinc-blende crystal structure yields the Dresselhaus or bulk inversion-asymmetry (BIA) contribution, $\boldsymbol{\Omega}_{\text {BIA }}$. A second contribution, the Rashba term $\boldsymbol{\Omega}_{\text {SIA }}$, results from structural inversion asymmetry (SIA) of the heterostructure, including the effect of an external applied electric field. ${ }^{3}$ A third contribution, which we denote as $\boldsymbol{\Omega}_{\mathbf{S T R}}$, and which is induced by the application of shear strain (STR) to the cubic zinc-blende structure ${ }^{6}$ is studied in detail in this paper. There is also a contribution induced by biaxial strain, which we do not consider here because it is known to be small compared to that from shear strain, although it is significant in other contexts. ${ }^{6,7}$ The different contributions in general each have a different dependence on $\mathbf{p}$ and can be varied independently of one another by the design of the heterostructure ${ }^{8-10}$ or by the application of external perturbations. ${ }^{11-14}$ Consequently, the interplay of the contributions that make up the resultant effective magnetic field, $\boldsymbol{\Omega}(\mathbf{p})=\boldsymbol{\Omega}_{\mathbf{B I A}}+\boldsymbol{\Omega}_{\mathbf{S I A}}+\boldsymbol{\Omega}_{\mathbf{S T R}}$, offers many possibilities for external control of the spin dynamics, including complete cancellation of one or more Cartesian components of $\boldsymbol{\Omega}(\mathbf{p}) .^{11,12,15}$
Spin rotation of a polarized electron population has been directly observed under a uniform imposed drift, ${ }^{7,16-18}$ under movement by a surface acoustic wave, ${ }^{19}$ and also for electrons at the Fermi momentum in a degenerate two-dimensional (2D) electron gas. ${ }^{20}$ The momentum-dependent spin precession is also the basis for the Dyakonov-Perel (DP) spin-dephasing mechanism; ${ }^{21}$ due to its random thermal motion, each electron senses a rapidly fluctuating effective field, which produces small random spin rotations between scattering events and results in the spin relaxation of a thermal electron population. The rate of relaxation for a particular component of the spin polarization, $i$, can be described by

$$
\Gamma_{i}^{s}=\left\langle\Omega_{\perp}^{2}\right\rangle \tau_{p}^{*} \quad \text { for } \tau_{p}^{*} \ll \frac{1}{\left|\Omega_{\perp}\right|},
$$

where $\left\langle\boldsymbol{\Omega}_{\perp}^{2}\right\rangle$ is the mean-square component of $\boldsymbol{\Omega}(\mathbf{p})$ in the plane perpendicular to the direction $i$, and $\tau_{p}^{*}$ is the momentum scattering time. This expression applies to bulk and isotropic systems and gives an intuitive understanding of the process in lower-symmetry situations such as heterostructures. In the latter, the spin-relaxation rate is strictly described by a tensor, which may have off-diagonal elements. ${ }^{22}$ The compelling motivation for this work is the fact that the measurement of the spin-relaxation rate provides a simple method to explore the control of $\boldsymbol{\Omega}(\mathbf{p})$ by strain. This is of particular interest in lowdimensional structures due to the ability to preconfigure $\boldsymbol{\Omega}(\mathbf{p})$. Furthermore, the dominance of the DP mechanism means that the spin-splitting vector $\boldsymbol{\Omega}(\mathbf{p})$ is the key to manipulation of spin memory.

We use spin quantum beat spectroscopy ${ }^{23,24}$ to investigate the effect on the spin dynamics of continuously tunable shear strain applied in the plane of an undoped, symmetric, 11.2-nm, (001)-oriented $\mathrm{GaAs} / \mathrm{Al}_{0.4} \mathrm{Ga}_{0.6}$ As quantum well. The method gives the spin-relaxation rates for spin components along the growth axis, $\Gamma_{\|}^{s}$, and in the quantum-well plane, $\Gamma_{\perp}^{s}$, and also the in-plane Landé $g$ factor of the photoexcited conduction electrons. We find that for zero strain, both $\Gamma_{\perp}^{s}$ and $g$ are 
isotropic. However, according to symmetry arguments, the strain may generate twofold anisotropy in both $\Gamma_{\perp}^{s}$ and $g$. While the applied strain induces significant anisotropy of $\Gamma_{\perp}^{s}$, we find no measurable anisotropy of the $g$ factor.

\section{THEORY}

The measured (001)-oriented quantum wells with symmetrical confinement potential and no applied electric field have the point symmetry $D_{2 d} \cdot{ }^{3}$ In the zinc-blende structure, shear strain has the same symmetry transformation properties as a vector, i.e., shear strain in the $x y$ plane of the quantum well, $\epsilon_{x y}$, transforms as a $z$-oriented vector and is, from a symmetry standpoint, equivalent to an electric field along the growth axis; both perturbations reduce the point symmetry from $D_{2 d}$ to $C_{2 v}$. For $C_{2 v}$ point symmetry, both $\Gamma_{\perp}^{s}$ and $g$ may acquire a twofold in-plane anisotropy. However, the observed anisotropy and its strength depend on the microscopic mechanisms involved. ${ }^{25}$ In the case of $\Gamma_{\perp}^{s}$, the application of strain induces an STR component of the spin-splitting vector, which interferes with the intrinsic BIA component and generates the anisotropy. This is exactly analogous to the interference of SIA and BIA components for an applied electric field, as predicted originally by Averkiev and Golub. ${ }^{10}$ SIA spin splitting reflects the coupled motion of electrons and holes, and it has been shown that when these motions are decoupled, the splitting is proportional to the expectation value of the valence-band potential gradient over the conduction electron wave function. ${ }^{3,26}$ Thus, from symmetry considerations, we may expect that the STR component of the splitting is similarly determined by the expectation value of an effective valenceband potential gradient related to the applied shear strain. In contrast, for the $g$ factor in (001)-oriented quantum wells, in-plane anisotropy is directly proportional to the asymmetry of the conduction electron envelope function. ${ }^{27,28}$ Such an asymmetry is incompatible with $D_{2 d}$ point symmetry, but is allowed in $C_{2 v}$, and can be induced by a gradient of the conduction-band potential due to an applied electric field or strain. Thus, the anisotropies of $\Gamma_{\perp}^{s}$ and $g$ have different microscopic mechanisms and give insight into the effect of the strain on the band edges of the heterostructure.

In the experiments described here, the DP spinrelaxation rate in Eq. (1) is proportional to $\left\langle\boldsymbol{\Omega}^{2}\right\rangle$, where $\boldsymbol{\Omega}=\boldsymbol{\Omega}_{\mathrm{BIA}}(\mathrm{p})+\boldsymbol{\Omega}_{\mathrm{STR}}(\mathrm{p})$, and to the lowest order in the electron momentum,

$$
\boldsymbol{\Omega}_{\mathrm{BIA}}(\mathrm{p})=\frac{\beta}{\hbar^{2}}\left(\begin{array}{c}
-p_{x} \\
p_{y} \\
0
\end{array}\right), \quad \boldsymbol{\Omega}_{\mathrm{STR}}(\mathrm{p})=\frac{\eta}{\hbar^{2}}\left(\begin{array}{c}
p_{y} \\
-p_{x} \\
0
\end{array}\right),
$$

with

$$
\beta=\left\langle p_{z}^{2}\right\rangle \gamma / \hbar^{2}
$$

where $\gamma$ is known as the Dresselhaus coefficient, and

$$
\eta=C_{3} \epsilon_{x y}
$$

where $C_{3}$ is a constant. The axes $x, y$, and $z$ refer to the cubic axes of the zinc-blende structure. (Note that the form of the STR component is the same as that of the SIA component if $\epsilon_{x y}$ is replaced by electric field $E_{z}$ and $C_{3}$ by the Rashba coefficient.) Both components of $\boldsymbol{\Omega}$ are oriented in the quantum-well plane, but their interference results in a twofold anisotropic spin-relaxation rate. ${ }^{10}$

We can see from the work of Averkiev and Golub ${ }^{10}$ that the DP relaxation rate derived from Eq. (1) for a spin component making an angle $\varphi$ to the (110) axis in the quantum-well plane is

$$
\Gamma_{\perp}^{s}(\varphi)=\frac{C}{2}\left[\eta^{2}+\beta^{2}-2 \eta \beta \cos (2 \varphi)\right],
$$

where $C$ is a constant determined by the momentum scattering time, $\tau_{p}^{*}$. They also showed that the relaxation rate for the spin component along the growth direction is

$$
\Gamma_{\|}^{s}=C\left(\eta^{2}+\beta^{2}\right) .
$$

In our experiment, the spins are initialized along the growth axis and a magnetic field is applied in the quantum-well plane, causing rapid Larmor precession of the spins about the field. We measure the dynamics of the $z$ component of the electron spins for the magnetic field at angle $\theta$ to (110) giving the electron Landé $g$ factor and the relaxation rate. The latter is taken to be the average $1 / 2\left[\Gamma_{\|}^{s}+\Gamma_{\perp}^{s}(\varphi)\right]$. If the $g$ factor is isotropic in the plane of the quantum well, as turns out to be the case for these measurements, then the spins precess in a plane perpendicular to the magnetic field and the measured spin-relaxation rate projected on the growth axis becomes

$$
\begin{aligned}
\Gamma^{s}(\theta) & =\frac{1}{2}\left[\Gamma_{\|}^{s}+\Gamma_{\perp}^{s}\left(\theta+\frac{\pi}{2}\right)\right] \\
& =\frac{3 C \beta^{2}}{4}\left[1+\left(\frac{\eta}{\beta}\right)^{2}+\frac{2 \eta}{3 \beta} \cos (2 \theta)\right] .
\end{aligned}
$$

In $D_{2 d}$, the $g$ tensor has two independent components, i.e., $g_{x x}=g_{y y}$ and $g_{z z}$. The reduction of the symmetry to $C_{2 v}$ allows in-plane anisotropy of the $g$ factor, which can be specified by nonzero off-diagonal elements of the $g$ tensor, ${ }^{25,28}$ $g_{x y}=g_{y x} \neq 0$. Thus,

$$
g(\theta)=-\sqrt{g_{x x}^{2}+g_{x y}^{2}+2 g_{x x} g_{x y} \cos (2 \theta)},
$$

where $\theta$ is the angle of the magnetic field in the quantum-well plane with respect to the (110) direction. For the width of the quantum well used here, it is known that $g_{x x}$ is negative, ${ }^{23}$ which is the origin of the minus sign in Eq. (5). Microscopic analysis gives ${ }^{27}$

$$
g_{x y}=g_{y x}=\left(\frac{2 \gamma e}{\hbar^{3} \mu_{B}}\right)\left(\left\langle p_{z}^{2}\right\rangle\langle z\rangle-\left\langle p_{z}^{2} z\right\rangle\right),
$$

where \langle\rangle denotes an expectation value over the electron wave function. The second bracket vanishes if the electron wave function is symmetrical with respect to the reflection in a plane perpendicular to the growth direction. Thus, the $g$-factor anisotropy in the plane is determined by the asymmetry of the electron wave function, and the prefactor contains the Dresselhaus coefficient $\gamma$.

\section{EXPERIMENT}

For the application of strain in the quantum-well plane, we used the technique developed by Shayegan et al. ${ }^{29}$ The substrate of the sample was first thinned to $\approx 100 \mu \mathrm{m}$ and subsequently glued to one side of a $\mathrm{PbZrTiO}_{3}$ (PZT) piezo 
stack using a specially formulated epoxy resin. A T-Rosette strain gauge was attached to the opposite side of the transducer to measure simultaneously the strains along the two transverse axes of the stack. The application of voltage produced lengthways expansion accompanied by transverse contraction; the ratio of longitudinal to transverse strain was $\approx-2$. In a separate test of this method of applying strain using a similar piezo and a dummy sample, we found that the strain recorded by a strain gauge attached directly to the piezo stack was very close to that recorded by a second strain gauge glued to the top of the dummy sample, indicating that the strain was efficiently transmitted to the quantum wells. This reproduced equivalent tests reported by Shayegan et al. For this experiment, the sample was oriented, to maximize shear strain, with cubic axes at 45 degrees to the axes of the stack. We define the direction of tensile strain to be the (110) axes, as shown in the inset of Fig. 1.

The sample-transducer arrangement was mounted on a rotation stage in a liquid-helium magnet cryostat. The growth axis of the sample coincided with the rotation axis of the mount and was aligned perpendicular to the horizontal, $5 \mathrm{~T}$ magnetic field. A mode-locked Ti:Sapphire laser with $80 \mathrm{MHz}$ repetition frequency, 2 ps pulse length, tuned to $750 \mathrm{~nm}$ and aligned parallel to the growth direction was used to excite photoluminescence (PL) from the sample. The light was circularly polarized and focused onto the sample with an excitation density of $\approx 1 \times 10^{9} \mathrm{~cm}^{-2}$. The PL was collected along the sample growth axis and analyzed using a liquidcrystal retarder and a polarizer to record the two circular polarization components consecutively. These components are spectrally and temporally resolved using a synchroscan streak camera imaging system with resolution of $0.5 \mathrm{~nm}$ and $8 \mathrm{ps,}$ respectively. Absorption of the circularly polarized light creates spin-polarized populations of electrons and holes, whose momentum distribution rapidly thermalizes after excitation via the emission of phonons and other scattering events. The hole population loses its spin orientation on the time scale of momentum relaxation ( $\tau_{p}^{*}<1 \mathrm{ps}$ ) due to strong valence-band mixing and momentum-dependent spin splitting. The degree

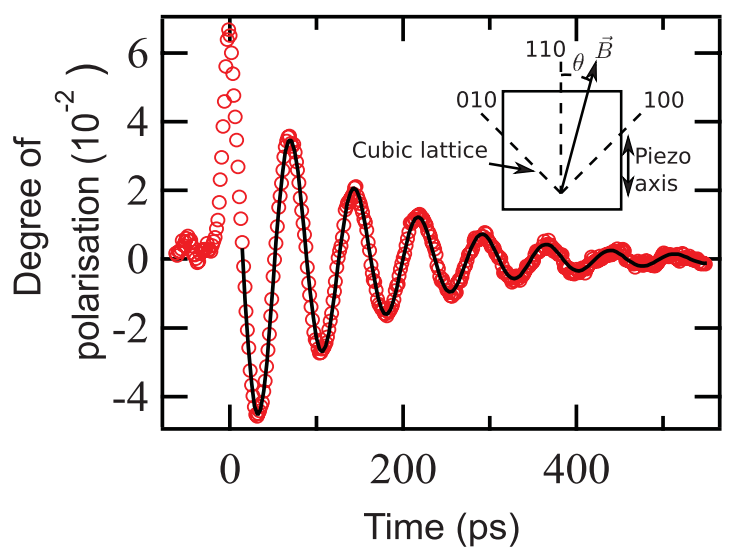

FIG. 1. (Color online) A typical decay curve (open circles) with a 5-T magnetic field oriented at $\theta=90^{\circ}$, at a temperature of $180 \mathrm{~K}$ and an applied strain of $\epsilon_{x y}=0.0021$. The solid curve is a fit of $A \cdot \cos \left(\omega_{L t}\right) e^{-\Gamma^{s} t}$, as discussed in the text. of circular polarization of the PL, $\left(I_{\sigma^{+}}-I_{\sigma^{-}}\right) /\left(I_{\sigma^{+}}+I_{\sigma^{-}}\right)$, is thus equal to the conduction electron-spin polarization. ${ }^{23}$

Figure 1 shows a typical time evolution of the polarization measured at a temperature of $180 \mathrm{~K}$ for a strain of $\epsilon_{x y}=$ 0.0021 , which is the maximum value achievable at this temperature. The strain available from the piezo transducer at a given applied voltage falls markedly as the temperature is reduced, while the PL signal increases. All of the measurements we report here were conducted at $180 \mathrm{~K}$, which represents a compromise between maximal strain and a satisfactory signal-to-noise ratio of the PL. The observed oscillation of the degree of circular polarization is caused by the coherent Larmor precession of the conduction electron-spin population with frequency $\omega_{L}=\left(g \mu_{B} / \hbar\right) B$ about the external magnetic field $B$. In order to extract both the spin-relaxation rate $\Gamma^{s}$ and the $g$ factor, the data is fitted to $A \cdot \cos \left(\omega_{L} t\right) e^{-\Gamma^{s} t}$, with $A, \omega_{L}$, and $\Gamma^{s}$ as free parameters.

\section{DISCUSSION}

Figure 2 shows the measured spin-relaxation rate (filled circles) and $g$ factor (open circles) for zero strain and for $\epsilon_{x y}=0.0021$ plotted versus the angle of the magnetic field with respect to the (110) in-plane crystal axis. The curves in Fig. 2 show the fits of Eqs. (4) and (5) for $\Gamma^{s}$ and $g$, which give values of $g_{x y}, g_{x x}$ and $\eta / \beta$ for different values of applied strain. The value of $\eta$ was extracted by calculating $\beta$ from Eq. (2), taking the value of $\gamma$ for this well width to be $9.5 \mathrm{eV} \AA^{3}$ from
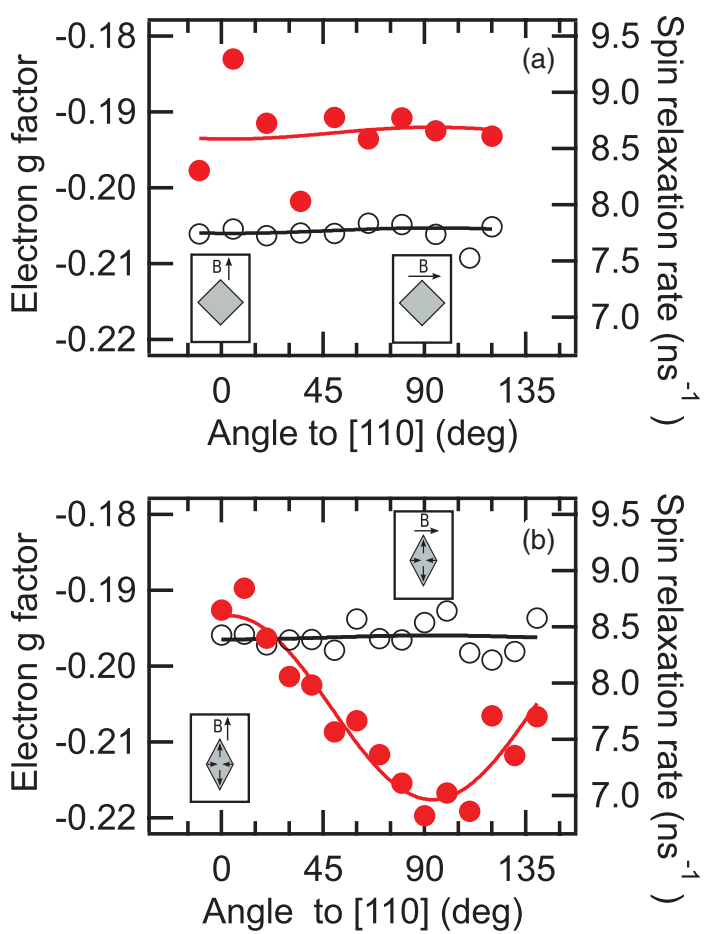

FIG. 2. (Color online) Dependence of the electron $g$ factor (open circles) and the spin-relaxation rate (solid circles) on the magneticfield orientation. (a) Zero applied strain and (b) a shear strain $\epsilon_{x y}=$ 0.0021 . The insets show the orientation of the sample with respect to the magnetic field $B$. In both plots, the insets indicate the compressive strain along (110) and tensile strain along (110). 


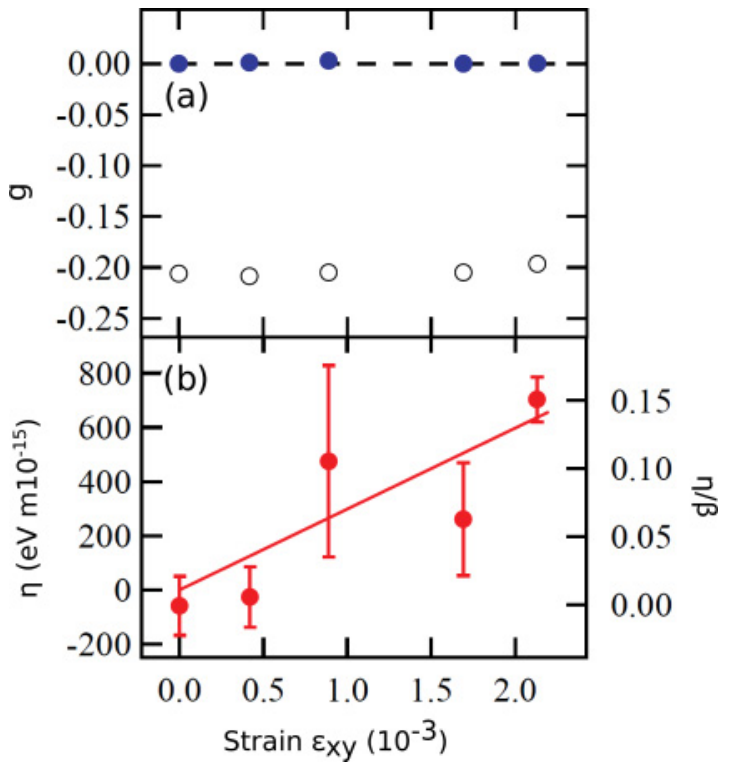

FIG. 3. (Color online) Variation with strain $\epsilon_{x y}$ of (a) $g_{x y}$ (solid circles) and $g_{x x}$ (open circles) and (b) $\eta$ and $\eta / \beta$. The gradient of linear fit (line) to the points in (b) gives $C_{3} / \hbar=4.7 \pm 1.1 \times 10^{5}$ $\mathrm{ms}^{-1}$.

Ref 20. Figure 3 shows these parameters plotted versus the applied strain.

For zero strain [Fig. 2(a)], neither quantity shows a detectable anisotropy, as is expected for an ideal quantum well with $D_{2 d}$ symmetry. When strain is applied [Fig. 2(b)], the spin-relaxation rate develops a significant twofold anisotropy, which is of the same form as that observed for the application of an electric field along the growth direction, confirming the symmetry argument given in Sec. II. The insets of Fig. 2(b) indicate the relative orientations of the magnetic field and the shear strain $\epsilon_{x y}$ in the sample; at 0 (90) degrees, the magnetic field is oriented parallel (at right angles) to the applied tension. From the figure, one can see that the extension of the sample parallel to the applied field increases the measured relaxation rate. This corresponds to an increase of the component of $\Omega$ in the direction of the tensile strain, i.e., $\Omega_{(110)}$ as defined here. Even for the highest value of applied strain, the $g$ factor remains isotropic within the experimental uncertainties. This scenario is the direct complementary case to the pure $g$-factor anisotropy without anisotropy of spin relaxation in a sample with asymmetric alloy composition, but where the bands are isomorphous, as discussed in Ref. 28. Calculations based on Eq. (6) show that an applied electric field of $\approx 10 \mathrm{kV} \mathrm{cm}^{-1}$ would be required to generate an anisotropy of the $g$ factor detectable above the uncertainties in Fig. 2. This places an upper limit on the conduction-band potential gradient generated by the maximum value of the applied strain in these measurements. It can be seen in Figs. 2 and 3(a) that the absolute value of the $g$ factor, $g_{x x}$, is slightly reduced by the applied strain. This trend is probably caused by an increase of the electron confinement energy, as indicated by the observed increase in the photoluminescence energy. The variation of $\eta$ with strain [Fig. 3(b)] gives the value of the strain spin-splitting coefficient, $C_{3} / \hbar=4.7 \pm 1.1 \times 10^{5} \mathrm{~ms}^{-1}$, in our 11.2-nm quantum-well sample. This value is somewhat less than the $8 \times 10^{5} \mathrm{~ms}^{-1}$ reported by Pikus et al. for bulk GaAs. ${ }^{6}$ It seems most likely that the difference represents a reduction of $C_{3}$ associated with quantum confinement of the electrons and may be described by $\mathbf{k} \cdot \mathbf{p}$ theory, as is the case for other spin-splitting coefficients, i.e., the electron $g$ factor ${ }^{3}$ and Dresselhaus coefficient $\gamma{ }^{20}$

Combined with the theoretical expectations outlined in Sec. II, the results lead to the following conclusions regarding the effect of strain on the band structure. First, Fig. 3(a) shows that within the experimental error, $g_{x y}$ is essentially zero for all values of strain; the average is $0.0005 \pm 0.015$. Referring to Eq. (6), this indicates that there is no detectable asymmetry of the electron wave function and, for this to be the case, the conduction-band potential remains, in good approximation, symmetrical up to the largest strain we can apply. Second, the observed linear strain-induced spin splitting indicated by the behavior of $\eta$ [Fig. 3(b)] shows that the strain acts primarily to generate an effective potential gradient in the valence band, which gives a nonzero expectation value over the electron wave function. It seems likely that this is associated with the fact that shear strain will not modify the $\Gamma_{6}$ conduction states, while it has some impact on the $\Gamma_{8}$ valence states.

\section{CONCLUSIONS}

We have used spin quantum beat spectroscopy to investigate the effect of in-plane shear strain on the conduction-band spin splitting and Larmor $g$ factor in undoped (001)-grown symmetric GaAs/AlGaAs quantum wells of width $11.2 \mathrm{~nm}$. The strain has the same symmetry transformation as a vector along the growth axis and reduces the point group symmetry from $D_{2 d}$ to $C_{2 v}$. The in-plane spin-relaxation rate becomes anisotropic, indicating a linear strain-induced contribution to the spin splitting. At its maximum, the measured value of $\Omega_{\mathrm{STR}}$ is $15 \%$ of $\Omega_{\mathrm{BIA}}$, indicating that in this sample, an approximately six-times-greater strain would be required to produce cancellation of $\Omega_{(1 \overline{1} 0)}$ and hence of $\Gamma_{(110)}^{s}$. Such a cancellation might be achieved in a sample with wider quantum wells, as $\Omega_{\text {BIA }}$ varies linearly with the electron confinement energy. In contrast, the in-plane $g$ factor remains isotropic within experimental uncertainties, although anisotropy is permitted by the strain-induced symmetry reduction.

The contrasting behavior of the spin-relaxation rate and the $g$ factor highlights their fundamental differences, although each is determined ultimately by the spin-orbit interaction. For the former, a nonzero expectation value of an effective valence-band potential gradient on the conduction-band states is required, whereas the latter requires the conduction-band electron wave function to be asymmetric. It is therefore clear from the experiment that strain in a GaAs/AlGaAs quantum well distorts the valence-band potential and so causes a spin splitting, but the conduction band and the wave function of the electron in the conduction band remain, in good approximation, symmetric.

\section{ACKNOWLEDGMENTS}

We acknowledge the financial support of SEPNET and EPSRC, the DFG priority program "SPP 1285-Semiconductor Spintronics," and the excellence cluster "QUEST." 
*Also at FORTH-IESL, P.O. Box 1385, 71110 Heraklion, Crete, Greece.

${ }^{1}$ S. Datta and B. Das, Appl. Phys. Lett. 56, 665 (1990).

${ }^{2}$ J. Schliemann, J. C. Egues, and D. Loss, Phys. Rev. Lett. 90, 146801 (2003).

${ }^{3}$ R. Winkler, Spin-Orbit Coupling Effects in Two-Dimensional Electron and Hole Systems, Springer Tracts in Modern Physics (Springer, Berlin, 2003).

${ }^{4}$ F. Meier and B. P. Zakharchenya, Optical Orientation, Modern Problems in Condensed Matter Sciences (Elsevier, New York, 1984), Vol. 8.

${ }^{5}$ M. I. Dyakonov, Spin Physics in Semiconductors, Springer Series in Solid-State Sciences (Springer, Berlin, 2008).

${ }^{6}$ G. E. Pikus, V. A. Marushchak, and A. N. Titkov, Sov. Phys. Semicond. 22, 115 (1988).

${ }^{7}$ B. M. Norman, C. J. Trowbridge, J. Stephens, A. C. Gossard, D. D. Awschalom, and V. Sih, Phys. Rev. B 82, 081304 (2010).

${ }^{8}$ M. I. Dyakonov and V. Y. Kachorovskii, Sov. Phys. Semicond. 20, 110 (1986).

${ }^{9}$ Y. Ohno, R. Terauchi, T. Adachi, F. Matsukura, and H. Ohno, Phys. Rev. Lett. 83, 4196 (1999).

${ }^{10}$ N. S. Averkiev and L. E. Golub, Phys. Rev. B 60, 15582 (1999).

${ }^{11}$ S.-W. Chang and S.-L. Chuang, Phys. Rev. B 72, 115429 (2005).

${ }^{12}$ X. Cartoixà, D. Z.-Y. Ting, and Y.-C. Chang, Phys. Rev. B 71, 045313 (2005).

${ }^{13}$ O. Z. Karimov, G. H. John, R. T. Harley, W. H. Lau, M. E. Flatté, M. Henini, and R. Airey, Phys. Rev. Lett. 91, 246601 (2003).

${ }^{14}$ A. V. Larionov and L. E. Golub, Phys. Rev. B 78, 033302 (2008).

${ }^{15}$ A. Balocchi, Q. H. Duong, P. Renucci, B. Liu, C. Fontaine, T. Amand, D. Lagarde, and X. Marie, Phys. Rev. Lett. 107, 136604 (2011).
${ }^{16}$ Y. Kato, R. C. Myers, A. C. Gossard, and D. D. Awschalom, Nature (London) 427, 50 (2004).

${ }^{17}$ S. A. Crooker and D. L. Smith, Phys. Rev. Lett. 94, 236601 (2005).

${ }^{18}$ M. Studer, M. P. Walser, S. Baer, H. Rusterholz, S. Schön, D. Schuh, W. Wegscheider, K. Ensslin, and G. Salis, Phys. Rev. B 82, 235320 (2010).

${ }^{19}$ J. A. H. Stotz, R. Hey, P. V. Santos, and K. H. Ploog, Nature Mater. 4, 585 (2005).

${ }^{20}$ W. J. H. Leyland, R. T. Harley, M. Henini, A. J. Shields, I. Farrer, and D. A. Ritchie, Phys. Rev. B 76, 195305 (2007).

${ }^{21}$ M. Dyakonov and V. Perel, Sov. Phys. Solid State 13, 3023 (1972).

${ }^{22}$ S. A. Tarasenko, Phys. Rev. B 80, 165317 (2009).

${ }^{23}$ M. Oestreich, S. Hallstein, and W. W. Ruhle, IEEE J. Sel. Top. Quantum Electron. 2, 747 (1996).

${ }^{24}$ J. Hübner and M. Oestreich, in Spin Physics in Semiconductors, edited by M. I. Dyakonov, Springer Series in Solid-State Sciences (Springer, Berlin, 2008), Chap. 5, pp. 211-244.

${ }^{25}$ J. Hübner, S. Kunz, S. Oertel, D. Schuh, M. Pochwała, H. T. Duc, J. Förstner, T. Meier, and M. Oestreich, Phys. Rev. B 84, 041301 (2011).

${ }^{26}$ P. S. Eldridge, W. J. H. Leyland, P. G. Lagoudakis, R. T. Harley, R. T. Phillips, R. Winkler, M. Henini, and D. Taylor, Phys. Rev. B 82, 045317 (2010).

${ }^{27}$ V. K. Kalevich and V. L. Korenev, JETP Lett. 57, 571 (1993).

${ }^{28}$ P. S. Eldridge, J. Hübner, S. Oertel, R. T. Harley, M. Henini, and M. Oestreich, Phys. Rev. B 83, 041301 (2011).

${ }^{29}$ M. Shayegan, K. Karrai, Y. P. Shkolnikov, K. Vakili, E. P. De Poortere, and S. Manus, Appl. Phys. Lett. 83, 5235 (2003). 